\title{
Singularity Theorems in General Relativity: Achievements and Open Questions.
}

\author{
José M. M. Senovilla \\ Física teórica, Universidad del País Vasco, Apartado 644, \\ 48080 Bilbao, Spain, josemm. senovilla@ehu.es
}

\section{Introduction}

In this short note, written by a theoretical physicist, not a historian, I would like to present a brief overview of the acclaimed singularity theorems, which are often quoted as one of the greatest theoretical accomplishments in General Relativity and Mathematical Physics.

Arguably, the singularity theorems are one of the few, if not the only one, consequences of Einstein's greatest theory which was not foreseen, probably not even suspected, by its founder. Many other consequences came also to a scientific firm basis only after Einstein's demise; to mention a few outstanding examples, let me cite gravitational lensing, gravitational radiation in binary systems (as in the PSR B1913+16 system), or the variation of frequency in electromagnetic radiation travelling in a gravity field. All of them, however, had been explicitly predicted in a way or another by Einstein.

On the contrary, the singularity theorems such as we understand them now (the result in (Einstein, 1941) is concerned with quite another type of singularities), the global developments needed for them, and the derived inferences, were not mentioned, neither directly nor indirectly, in any of his writings. This was so despite the various clear indications that the appearance of some kind of "singularity", or catastrophical behaviour, was a serious possibility within the orthodox theory. For instance, the FriedmanLemaître models (Friedman, 1922, 1924; Lemaitre, 1927), generally accepted by the 1930's as providing explanation for the observed galactic (or nebulosae) redshifts, contain the famous "creation time" (Friedman's wording) 
identified by Friedman and Lemaitre themselves, at which the space-time is simply destroyed. Or even grosser, the proof by Oppenheimer and Snyder (Oppenheimer and Snvder, 1939) that the "Schwarzschild surface" - which, as we know, is not a singularity, but a horizon, see for instance the well-

documented historical review on this subject presented in (Tipler. Clarke and Ellis, 1980) - was reachable and crossable by innocuous models containing realistic matter such as dust. (By the way, this dust eventually ends in a catastrophic (real) future singularity.) Not to mention the impressive result found by Chandrasekhar - to which Eddington opposed furiously - of the upper mass limit for stars in equilibrium, even when taking into account the quantum effects, implying that stars with a larger mass will inevitably collapse (Chandrasekhar, 1931).

In spite of all these achievements, as I was saying, Einstein and the orthodoxy simply dismissed the catastrophic behaviours (singularities) as either a mathematical artifact due to the presence of (impossible) exact spherical symmetry, or as utterly impossible effects, scientifically untenable, obviously unattainable, beyond the feasibility of the physical world - see e.g. (Einstein, 1939). Of course, this probably is certain in a deep sense, as infinite values of physical observables must not be accepted and every sensible scientist would defend similar sentences. However, one must be prepared to probe the limits of any particular theory, and this was simply not done with General Relativity at the time. It was necessary to wait for a new generation of physicists and mathematicians, without the old prejudices and less inhibited, and probably also less busy with the quantum revolution, who could finally take the question of the singularities, the gravitational collapse, and the past of the Universe, seriously within the theory.

Thus, one can say that the singularity theorems are the most genuine post-Einsteinian content of General Relativity.

\subsection{The Raychaudhuri equation}

Curiosuly enough, the first result concerning prediction of singularities under reasonable physical conditions, due to Raychaudhuri, came to light exactly the same year of Einstein's decease. In 1955 Raychaudhuri published what is considered the first singularity theorem, and included a version of the equation named after him which is the basis of later developments and of all the singularity theorems (Ravchaudhuri, 1955). The Raychaudhuri equation can be easily derived (see for instance (Dadhich, 2005) in a recent tribute to 
Raychaudhuri), as it has a very simple geometrical interpretation: take the well-known Ricci identity

$$
\left(\nabla_{\mu} \nabla_{\nu}-\nabla_{\nu} \nabla_{\mu}\right) u^{\alpha}=R_{\rho \mu \nu}^{\alpha} u^{\rho}
$$

which is mathematically equivalent to the standard definition of the Riemann tensor

$$
\left(\nabla_{\vec{X}} \nabla_{\vec{Y}}-\nabla_{\vec{Y}} \nabla_{\vec{X}}-\nabla_{[\vec{X}, \vec{Y}]}\right) \vec{Z}=R(\vec{X}, \vec{Y}) \vec{Z} \quad \forall \vec{X}, \vec{Y}, \vec{Z}
$$

and contract $\alpha$ with $\mu$ in (11), then with $u^{\nu}$, to obtain

$$
u^{\nu} \nabla_{\mu} \nabla_{\nu} u^{\mu}-u^{\nu} \nabla_{\nu} \nabla_{\mu} u^{\mu}=R_{\rho \nu} u^{\rho} u^{\nu}
$$

where $R_{\rho \nu}$ is the Ricci tensor. Reorganizing by parts the first summand on the lefthand side one derives

$$
u^{\nu} \nabla_{\nu} \nabla_{\mu} u^{\mu}+\nabla_{\mu} u_{\nu} \nabla^{\nu} u^{\mu}-\nabla_{\mu}\left(u^{\nu} \nabla_{\nu} u^{\mu}\right)+R_{\rho \nu} u^{\rho} u^{\nu}=0
$$

which is the Raychaudhuri equation. Raychaudhuri's important contribution amounts to understanding the physical implications of this relation. Observe that, in the case that $u^{\mu}$ defines a (affinely parametrized) geodesic vector field, then $u^{\nu} \nabla_{\nu} u^{\mu}=0$ and the third term vanishes. The second term can be rewritten by splitting

$$
\nabla_{\mu} u_{\nu}=S_{\mu \nu}+A_{\mu \nu}
$$

into its symmetric $S_{\mu \nu}$ and antisymmetric $A_{\mu \nu}$ parts, so that

$$
\nabla_{\mu} u_{\nu} \nabla^{\nu} u^{\mu}=S_{\mu \nu} S^{\mu \nu}-A_{\mu \nu} A^{\mu \nu}
$$

Now the point is to realize that (i) if $u^{\mu}$ is time-like (and normalized) or null, then both $S_{\mu \nu} S^{\mu \nu}$ and $A_{\mu \nu} A^{\mu \nu}$ are non-negative; and (ii) $u_{\mu}$ is proportional to a gradient (therefore defining orthogonal hypersurfaces) if and only if $A_{\mu \nu}=0$. In summary, for hypersurface-orthogonal geodesic time-like or null vector fields $u^{\mu}$ one has

$$
u^{\nu} \nabla_{\nu} \nabla_{\mu} u^{\mu}=-S_{\mu \nu} S^{\mu \nu}-R_{\rho \nu} u^{\rho} u^{\nu}
$$

so that the sign of the derivative of the divergence $\nabla_{\mu} u^{\mu}$ along these geodesics is governed by the sign of $R_{\rho \nu} u^{\rho} u^{\nu}$. If the latter is non-negative, then the 
former is non-positive. In particular, if the divergence is negative at some point and $R_{\rho \nu} u^{\rho} u^{\nu} \geq 0$ then necessarily the divergence will reach an infinite negative value in finite affine parameter (unless all the quantities are zero everywhere).

If there are physical particles moving along these geodesics, then clearly a physical singularity is obtained, as the average volume decreases and the density of particles will be unbounded. This was the situation treated by Raychaudhuri for the case of irrotational dust. In general, no singularity is predicted, though, and one only gets a typical caustic along the flow lines of the congruence defined by $u^{\mu}$. This generic property is usually called the focusing effect on causal geodesics. For this to take place, of course, one needs the condition

$$
R_{\rho \nu} u^{\rho} u^{\nu} \geq 0
$$

which is a geometric condition and independent of the particular theory. However, in General Relativity, one can relate the Ricci tensor to the energymomentum tensor $T_{\mu \nu}$ via Einstein's field equations

$$
R_{\mu \nu}-\frac{1}{2} g_{\mu \nu} R+\Lambda g_{\mu \nu}=\frac{8 \pi G}{c^{4}} T_{\mu \nu}
$$

where $R$ is the scalar curvature, $G$ is Newton's gravitational constant, $c$ is the speed of light in vacuum and $\Lambda$ the cosmological constant. Thereby, the condition (3) can be rewritten in terms of physical quantities. This is why sometimes (3), when valid for all time-like $u^{\mu}$, is called the strong energy condition (Hawking and Ellis, 1973). One should bear in mind, however, that this is a condition on the Ricci tensor (a geometrical object) and therefore it will not always hold: see the discussion in Section 3 below and (Senovilla, 1998a, sect. 6.2).

An important remark of historical importance is that, before 1955, Gödel wrote his famous paper (Gödel, 1949) in a volume dedicated to Einstein's 70th anniversary. This paper is considered (see Tipler. Clarke and Ellis, 1980, sect. 3) the genesis of many of the necessary techniques and some of the global ideas which were used in the path to the singularity theorems, specially concerning causality theory. For further information the reader is referred to (Ellis, 1998). However, the subject was not ripe and had to wait, first, to the contribution by Raychaudhuri, and then, to the imaginative and fruitful ideas put forward by Roger Penrose in the 1960s. 


\section{Remarks on singularities and extensions}

The problem of the definition of the concept of singularity in General Relativity is very difficult indeed, as can be appreciated by reading on its historical development (Hawking and Ellis, 1973; Tipler. Clarke and Ellis, 1980). The intuitive ideas are clear: if any physical or geometrical quantity blows up, this signals a singularity. However, there are problems of two kinds:

- the singular points, by definition, do not belong to the space-time which is only constituted by regular points. Therefore, one cannot say, in principle, "when" or "where" is the singularity.

- characterizing the singularities is also difficult, because the divergences (say) of the curvature tensor can depend on a bad choice of basis, and even if one uses only curvature invariants, independent of the bases, it can happen that all of them vanish and still there are singularities.

The second point is a genuine property of Lorentzian geometry, that is, of the existence of one axis of time of a different nature to the space axes.

Therefore, the only sensible definition with a certain consensus within the relativity community is by "signalling" the singularities, "pointing at them" by means of quantities belonging to the space-time exclusively. And the best and simplest pointers are curves, of course. One can imagine what happens if a brave traveller approaches a singularity: he/she will disappear from our world in a finite time. The same, but time-reversal, can be imagined for the "creation" of the Universe: things suddenly appeared from nowhere a finite time ago. All in all, it seems reasonable to diagnose the existence of singularities whenever there are particles (be them real or hypothetical) which go to, or respectively come from, them and disappear unexpectedly or, respectively, subito come to existence.

And this is the basic definition of singularity (Geroch, 1968; Hawking and Ellis, 1973), the existence of incomplete and inextensible curves. That is to say, curves which cannot be extended in a regular manner within the space-time and do not take all possible values of their canonical parameter. Usually, only causal (time-like or null) curves are used, but in principle also incomplete space-like curves define singularities. The curves do not need to be geodesic, and as a matter of fact there are known examples of geodesically complete space-times with incomplete time-like curves of everywhere bounded acceleration (Geroch, 1968). It must be remarked, however, that all singularity 
theorems prove merely the existence of geodesic incompleteness, which of course is a sufficient condition for the existence of singularities according to the definition.

Some fundamental questions, which sometimes are omitted, arise at this point. How can one give structure and properties to the singularities? What is the relation between geodesic incompleteness and curvature problems, if any? Singularities in the above sense clearly reach, or come from, the edge of space-time. This is some kind of boundary, or margin, which is not part of the space-time but that, somehow, it is accessible from within it. Thus the necessity of a rigurous definition of the boundary of a space-time. Given that a Lorentzian manifold is not a metric space in the usual sense, one cannot use the traditional completion procedure by means of Cauchy sequences. The most popular approach in this sense has been the attempt to attach a causal boundary to the space-time, see (García-Parrado and Senovilla, 2005) for an up-to-date review. But this is not exempt of recurrent problems which have not been completely solved yet.

Furthermore, the existence of incomplete geodesics which are not extensible in a given space-time may indicate not a problem with the curve and the geometrical properties along it when approaching the edge, but rather incompleteness of the space-time itself. For instance, flat space-time without the origin has infinite inextensible incomplete curves. These cases, however, are to be avoided, as one can easily see that the problem arises due to the excision of regular points. This is why one usually restricts considerations to inextensible space-times when dealing with singularity theorems (Hawking and Ellis, 1973). The physical problem, however, is hidden under the carpet with this attitude because: what are we supposed to do with given extensible space-times? The answer may seem simple and easy: just extend it until you cannot extend it anymore. However, this is not so simple for several reasons (see Senovilla, 1998a, sects. 3 and 7):

- extensions are not obvious, and generally not unique. Usually there are infinite inequivalent choices.

- not even analytical extensions are unique in general, let alone smooth extensions.

- it can happen that there are incomplete curves, no curvature problems, and no possible (regular) extension. 
- sometimes, for a given fixed extensible space-time, there are extensions leading to new extensible space-times, other extensions leading to singular space-times, and even other extensions which are free of singularities and inextensible. It may seem obvious that the last choice would be the preferred one by relativists, but this is simply not the case - if the singularity-free extension violates a physical condition, such as causality or energy positivity, then the other extensions will be chosen-.

- which physical reasons are to be used to discriminate between inequivalent extensions?

As a drastic example of the above, take the traditional case of the Schwarzschild solution which, as is known, is extensible through the horizon. In textbooks one usually encounters the unique maximal analytical vacuum extension due to Kruskal and Szekeres keeping spherical symmetry. However, if one drops any one of the conditions (vacuum, analyticity) many other maximal extensions are possible, see e.g. (Senovilla, 1998a) where at least eleven inequivalent extensions were explicitly given. This should make plain that the question of singularities is intimately related to the problem of how, why and to where a given extensible space-time must be extended.

\section{Singularity theorems: Critical appraisal}

The first singularity theorem in the modern sense is due to Penrose (Penrose, 1965), who in this seminal paper introduced the fundamental concept of closed trapped surface and opened up a new avenue of fruitful research. His main, certainly innovative idea, was to prove null geodesic incompleteness if certain initial conditions, reasonable for known physical states of collapse, were given irrespective of any symmetry or similar restrictive properties.

Since then, many singularity theorems have been proven (see Hawking and Ellis, 1973; Senovilla, 1998a), some of them applicable to cosmological situations, some to star or galaxy collapse, and others to the collision of gravitational waves. The culmination was the celebrated Hawking-Penrose theorem (Hawking and Penrose, 1970), which since then is the singularity theorem par excellence. However, all of the singularity theorems share a well-defined skeleton, the very same pattern. This is, succintly, as follows (Senovilla, 1998a) 
Theorem 1 (Pattern Singularity Theorem) If a space-time of sufficient differentiability satisfies

1. a condition on the curvature

2. a causality condition

3. and an appropriate initial and/or boundary condition

then there are null or time-like inextensible incomplete geodesics.

I have started by adding explicitly the condition of sufficient differentiability. This is often ignored, but it is important from both the mathematical and the physical points of view. A breakdown of the differentiability should not be seen as a true singularity, specially if the problem is mild and the geodesic curves are continuous. The theorems are valid if the space-time metric tensor field $g_{\mu \nu}$ is of class $C^{2}$ (twice differentiable with continuity), but they have not been proven in general if the first derivatives of $g_{\mu \nu}$ satisfy only the Lipshitz condition. This problem is of physical relevance, because the entire space-time of a star or a galaxy, say, is usually considered to have two different parts matched together at the surface of the body, and then the metric tensor is not $C^{2}$ at this surface: it cannot be, for there must exist a jump in the matter density which is directly related to the second derivatives of $g_{\mu \nu}$ via equations (4). As an example, the Oppenheimer-Snyder collapsing model does not satisfy the $C^{2}$ condition. A list of the very many places where this condition is used in the singularity theorems can be found in (Senovilla, 1998a, p. 799).

Then there is the "curvature condition". I have preferred this name rather than the usual energy and generic condition to stress the fact that this assumption is of a geometric nature, and it is absolutely indispensable: it enforces the geodesic focusing via the Raychaudhuri equation - and other similar identities - . The majority of the theorems, specially the stronger ones, use the condition (3), which is usually called strong energy condition if it is valid for all time-like $u^{\mu}$, and null convergence condition if it is valid only for null vectors. The former name is due to the equivalent relation, via the field equations (4)

$$
T_{\rho \nu} u^{\rho} u^{\nu} \geq \frac{c^{4} \Lambda}{8 \pi G}-\frac{1}{2} T^{\rho}{ }_{\rho}
$$


for unit time-like $u^{\mu}$. This involves energy-matter variables. However, this condition does not have to be satisfied in general by realistic physical fields. To start with, it depends on the sign of $\Lambda$. But furthermore, even if $\Lambda=0$, the previous condition does not hold in many physical systems, such as for instance scalar fields (Hawking and Ellis, 1973, p. 95). As a matter of fact, most of the inflationary cosmological models violate the above condition. Let us stress that the physically compelling energy condition is the dominant energy condition (Hawking and Ellis, 1973), but this has in principle nothing to do with the assumptions of the singularity theorems. In particular, there are many examples of reasonable singularity-free space-times satisfying the dominant, but not the strong, energy condition (see e.g. Senovilla, 1998a, sect.7.3). In my opinion, this is one of the weak points of the singularity theorems.

The "causality condition" is probably the most reasonable and wellfounded, and perhaps also the less restrictive, condition. There are several examples of singularity theorems without any causality condition (Hawking and Ellis, 1973, Theorem 4, p. 272) (Maeda and Ishibashi, 1996). The causality condition is assumed for two types of reasons. Firstly, to prevent the possibility of avoiding the future, that is to provide a well-defined global time-arrow. This may seem superfluous, but it is known since the results in (Gödel, 1949) that there may be closed time-like lines, that is, curves for which the time passes to the future permanently and nevertheless reach their own past. And secondly, to ensure the existence of geodesics of maximal proper time between two events, and therefore geodesics without focal points (Hawking and Ellis, 1973, Prop. 4.5.8).

Recapitulating, the first two conditions in the theorems imply

- the focusing of all geodesics, ergo the existence of caustics and focal points, due to the curvature condition

- the existence of geodesics of maximal proper time, and therefore necessarily without focal points, joining any two events of the space-time

Obviously, a contradiction starts to glimmer if all geodesics are complete. There is not such yet, though, and this is because at this stage there is no finite upper bound for the proper time of selected families of time-like geodesics (and analogously for null geodesics).

To get the final contradiction one needs to add the third condition. And this is why the initial/boundary condition is absolutely essential in the the- 
orems. There are several possibilities for this condition, among them (i) an instant of time at which the whole Universe is strictly expanding, (ii) closed universes so that they contain space-like compact slices; (iii) the existence of closed (that is, compact without boundary) trapped surfaces.

This last concept, due to Penrose (Penrose, 1965), has become the most popular and probably the most important one, specially due to its relevance an applicability in many other branches of Relativity. Trapped surfaces are 2-dimensional differentiable surfaces (such as a donut's skin or a sphere) with a mean curvature vector which is time-like everywhere, or in simpler words, such that the initial variation of area along any future direction is always decreasing (or always increasing). An example of a non-compact trapped surface in flat space-time (in Cartesian coordinates) is given by

$$
e^{t}=\cosh z, \quad x=\text { const. }
$$

There cannot be, however, compact trapped surfaces in flat space-time. An example of a compact trapped surface is given for instance by any 2-sphere $t=$ const., $x^{2}+y^{2}+z^{2}=R^{2}=$ const., in a Friedman model

$$
d s^{2}=-d t^{2}+a^{2}(t)\left(d x^{2}+d y^{2}+d z^{2}\right)
$$

as long as $R>1 /|\dot{a}|$, which is always possible.

Whether or not the initial or boundary condition is realistic, or satisfied by the actual physical systems, is debatable. We will probably find it very difficult to ascertain if the Universe is spatially finite, or if the whole Universe is strictly expanding now. There is a wide agreement, however, that it is at least feasible that in some situations the third condition in the theorems will hold. For example, given the extremely high degree of isotropy observed in the cosmic microwave background radiation, one can try to infer that the Universe resembles a Friedman-Lemaître model, thus containing trapped spheres as the one shown above. Nevertheless, there are several way outs to this scheme of reasoning, for example a cosmological constant, or deviations from the model at distances (or redshifts) of the order or higher than the visible horizon. For a discussion see (Senovilla, 1998a, sects. 6.4 and 7.2).

Let us finally pass to the conclusion of the theorems. In most singularity theorems this is just the existence of at least one incomplete causal geodesic. This is very mild, as it can be a mere localized singularity. This leaves one door open (extensions) and furthermore it may be a very mild singularity. In addition, the theorems do not say anything, in general, about the situation 
and the character of the singularity. We cannot know whether it is in the future or past, or whether it will lead to a blow-up of the curvature or not.

In the next section I am going to present a very simple solution of Einstein's field equations which shows explicitly the need of the "small print" in the theorems, and that sometimes their assumptions are more demanding than generally thought.

\section{An illustrative singularity-free space-time}

Nowadays there are many known singularity-free space-times, some of them are spatially inhomogeneous universes, others contain a cosmological constant, and there are a wide variety of other types (see Senovilla, 1998a, and references therein). Perhaps the most famous singularity-free "cosmological model" was presented in (Senovilla, 1990), because it had some impact on the scientific community (see e.g. Maddox, 1990) and opened up the door for many of the rest. The impact was probably due to the general belief that such a space-time was forbidden by the singularity theorems. But, of course, this was simply a misunderstanding, and it only meant that we had thought that the singularity theorems were implying more than they were actually saying.

The space-time has cylindrical symmetry, and in typical cylindrical coordinates $\{t, \rho, \varphi, z\}$ its line-element takes the form

$$
\begin{gathered}
d s^{2}=\cosh ^{4}(a c t) \cosh ^{2}(3 a \rho)\left(-c^{2} d t^{2}+d \rho^{2}\right)+ \\
\frac{1}{9 a^{2}} \cosh ^{4}(a c t) \cosh ^{-2 / 3}(3 a \rho) \sinh ^{2}(3 a \rho) d \varphi^{2}+\cosh ^{-2}(a c t) \cosh ^{-2 / 3}(3 a \rho) d z^{2}
\end{gathered}
$$

where $a>0$ is a constant. This is a solution of the field equations (44) for $\Lambda=0$ and an energy-momentum tensor of perfect-fluid type

$$
T_{\mu \nu}=\rho u_{\mu} u_{\nu}+p\left(g_{\mu \nu}+u_{\mu} u_{\nu}\right)
$$

where $\rho$ is the energy density of the fluid given by

$$
\frac{8 \pi G}{c^{4}} \varrho=15 a^{2} \cosh ^{-4}(a c t) \cosh ^{-4}(3 a \rho)
$$

$p$ its isotropic pressure and

$$
u_{\mu}=\left(-c \cosh ^{2}(a c t) \cosh (3 a \rho), 0,0,0\right)
$$


defines the unit velocity vector field of the fluid. Observe that $u^{\mu}$ is not geodesic (except at the axis). The fluid has a realistic barotropic equation of state

$$
p=\frac{1}{3} \rho .
$$

This is the canonical equation of state for radiation-dominated matter and is usually assumed at the early stages of the Universe. Note that the density and the pressure are regular everywhere, and one can in fact prove that the space-time is completely free of singularities and geodesically complete (Chinea. Fernández-Jambrina and Senovilla, 1992).

The space-time satisfies the stronger causality conditions (it is globally hyperbolic), and also all energy conditions (dominant, strict strong). The fluid divergence is given by

$$
\nabla_{\mu} u^{\mu}=3 a \frac{\sinh (a c t)}{\cosh ^{3}(a c t) \cosh (3 a \rho)}
$$

so that this universe is contracting for half of its history $(t<0)$ and expanding for the second half $(t>0)$, having a rebound at $t=0$ which is driven by the spatial gradient of pressure. Observe that the whole universe is expanding (that is, $\nabla_{\mu} u^{\mu}>0$ ) everywhere if $t>0$, and recall that this was one of the possibilities we mentioned for the third condition in the singularity theorems: an instant of time with a strictly expanding whole universe. Thus, how can this model be geodesically complete and singularity-free?

Well, the precise condition demanded by one of the theorems in globally hyperbolic space-times is that $\nabla_{\mu} u^{\mu}>b>0$ for a constant $b$. That is, $\nabla_{\mu} u^{\mu}$ has to be bounded from below by a positive constant. But this is not the case for (5), which is strictly positive everyhwere but not bounded from below by a positive constant because $\lim _{\rho \rightarrow \infty} \nabla_{\mu} u^{\mu}=0$. This minor, subtle, difference allows for the model to be singularity-free! All other possibilities for the initial/boundary condition in the several would-be applicable singularity theorems can be seen to just fail in the model, in a similar manner. For a complete discussion see (Chinea. Fernández-Jambrina and Senovilla, 1992) and (Senovilla, 1998a, sect. 7.6). One can also see that the focusing effect on geodesics takes place fully in this space-time, but nevertheless there is no problem with the existence of maximal geodesics between any pair of points (see Senovilla, 1998a, pp. 829-830).

This simple model showed that there exist well-founded, well-behaved classical models expanding everywhere, satisfying all energy and causality 
conditions, and singularity-free. Of course, the model is not realistic in the sense that it cannot describe the actual Universe - for instance, the isotropy of the cosmic background radiation cannot be explained-, but the question arises of whether or not there is room left over by the singularity theorems to construct geodesically complete realistic universes.

It should be stressed that this model does not describe a "cylindrical star", because the pressure of the fluid does not vanish anywhere. Nevertheless, as can be seen from the previous formulae, for example the energy density is mainly concentrated in an area around the axis of symmetry, dying away from it very quickly as $\rho$ increases. This may somehow put some doubts about the relevance of this type of models for cosmological purposes. In this sense, there was an interesting contribution by Raychaudhuri himself (Ravchaudhuri, 1998), where he tried to quantify this property in a mathematical condition. Unfortunately, this time he was not completely right (see Senovilla, 1998b), because he used space-time averages of the physical variables such as the energy density. But one can easily seen that the vanishing of such averages is a property shared by the majority of the models, be them singular or not. However, his work provided some inspiration, and I believe that the vanishing of the spatial averages of the physical variables is probably a condition which may allow to distinguish between the singularity-free models allowed by the singularity theorems and the singular ones. A conjecture of the singularity-theorem type was put forward in (Senovilla, 1998b) with this purpose.

All in all, the main conclusion of this contribution is to remind ourselves that it is still worth to develop further, understand better, and study careful the singularity theorems, and their consequences for realistic physical systems.

\section{References}

Chandrasekhar, Subrahmanyan (1931a) "The maximum mass of ideal white dwarfs" Astrophysical Journal 74 81-82

Chinea, F. Javier, Fernández-Jambrina, Leonardo and Senovilla, José M.M. (1992) "Singularity-free space-time" Physical Review D 45 481-486

Dadhich, Naresh (2005) "Derivation of the Raychaudhuri equation" gr-qc/0511123 
Einstein, Albert (1939) "On a stationary system with spherical symmetry consisting of many gravitating masses" Annals of Mathematics 40 922-936

(1941) "Demonstration of the non-existence of gravitational fields with a non-vanishing total mass free of singularitites" Revista de la Universidad Nacional de Tucumán A2 11-16

Ellis, George F.R. (1998) "Contributions of K. Gödel to Relativity and Cosmology". In Gödel '96: Foundations of Mathematics, Computer Science and Physics: Kurt Gödel's Legacy Lectures Notes in Logic 6, Petr Hajek ed. Berlin: Springer, 34-49

Friedman, Alexander (1922) "Über die Krümmung des Raumes" Zeitschrift für Physik 10 377-386;

Translated and reproduced in (1999) General Relativity and Gravitation 31 1991-2000

(1924) "Über die Möglichkeit einer Welt mit konstanter negativer Krümmung des Raumes" Zeitschrift für Physik 21 326-332;

Translated and reproduced in (1999) General Relativity and Gravitation 31 2001-2008

García-Parrado, Alfonso and Senovilla, José M.M. (2005), "Causal structures and causal boundaries" (Topical Review) Classical and Quantum Gravity 22 R1-R84

Geroch, Robert (1968) "What is a singularity in General Relativity" Annals of Physics (New York) 48 526-540

Gödel, Kurt (1949) "An example of a new type of cosmological solution of Einstein's field equations of gravitation" Reviews in Modern Physics 21 $447-450$

Hawking, Stephen W. and Ellis, Georges F.R. (1973) The Large Scale Structure of Space-time Cambridge: Cambridge University Press.

Hawking, Stephen W. and Penrose, Roger (1970) "The singularities of gravitational collapse and cosmology" Proceedings of the Royal Society London A314 529-548 
Lemaître, Georges (1927) "Un Universe homogène de masse constante et de rayon croissant, rendant compte de la vittese radiale des nébuleuses extragalactiques" Annales de la Société Scientifique de Bruxelles A47 4959

Maddox, John (1990) "Another gravitational solution found" Nature 345201

Maeda, Kengo and Ishibashi Akihiro (1996) "Causality violation and singularities" 13 2569-2576

Oppenheimer, Julius Robert and Snyder, Hartland (1939) "On continued gravitational contraction" Physical Review 56 455-459

Penrose, Roger (1965) "Gravitational collapse and space-time singularities" Physical Review Letters 14 57-59

Raychaudhuri, Amal Kumar (1955) "Relativistic Cosmology I" Physical Review 98 1123-1126

- (1998) "Theorem for Non-Rotating Singularity-Free Universes" Physical Review Letters 80 654-655

Senovilla, José M. M. (1990) "New class of inhomogeneous cosmological perfect-fluid solutions without big-bang singularity" Physical Review Letters 64 2219-2221

- (1998a) "Singularity Theorems and Their Consequences" (Review) General Relativity and Gravitation 30 701-848

(1998b) "Comment on 'Theorem for Non-Rotating Singularity-Free Universes' " Physical Review Letters $\mathbf{8 1} 5032$

Tipler, Frank J., Clarke, Chris J. S. and Ellis, George F. R. (1980) "Singularities and Horizons - A Review Article." In General Relativity and Gravitation: One Hundred Years After the Birth of Albert Einstein. Alan Held ed. New York: Plenum Press, 97-206 